\title{
Cue-induced transfer of CS preexposure effects across contexts
}

\author{
WILLIAM C. GORDON and MELANIE S. WEAVER \\ University of New Mexico, Albuquerque, New Mexico
}

\begin{abstract}
These experiments confirmed previous reports that latent inhibition effects can be abolished if rats receive CS preexposures and CS-US pairings in different contexts. However, latent inhibition effects were restored if animals were given a cuing treatment in the conditioning context prior to the conditioning trials. This restoration of latent inhibition occurred only when the cuing treatment could have served to remind the animals of CS-alone presentations in the specific context used for conditioning. These findings are discussed in relation to various accounts of latent inhibition effects.
\end{abstract}

It is well established that an animal's performance of a learned response is often context-dependent. In most cases, test performance tends to decrease the more dissimilar the test context is from the context present during learning (see Balsam \& Tomie, 1985). The particular mechanism by which context influences performance is currently the subject of much investigation.

According to one view (Rescorla \& Wagner, 1972), contextual stimuli function much like discrete conditioned stimuli (CSs) in Pavlovian conditioning situations. That is, contextual stimuli enter into associations with the unconditioned stimulus (US) at the time of learning and become capable of eliciting a conditioned response (CR). Thus, when animals undergo conditioning and testing in the same context, the contextual stimuli and the discrete CS combine to aid in the elicitation of the CR. However, when testing occurs in a novel context, only the CS is present to produce a response, and overall responding diminishes because of the absence of the learning context.

An alternative view of contextual effects (e.g., Medin, 1975; Nadel \& Willner, 1980; Spear, 1973) suggests that at the time of learning, contextual stimuli require conditional or retrieval cue status. In other words, during learning, contextual stimuli somehow become linked to the primary CS-US association or become encoded along with this association. Through this link, contextual stimuli become capable of retrieving an animal's memory of the CS-US association, making conditioned responding more probable. According to this interpretation, animals trained and tested in different contexts have greater difficulty retrieving the learned association at the time of testing than do animals trained and tested in the same context.

An assumption common to both of these positions is that contextual stimuli can come to control learned performance only to the extent that these stimuli are present at the time of learning. However, work carried out in our laboratory over the past several years has called this as-

Requests for reprints may be sent to William C. Gordon, Department of Psychology, University of New Mexico, Albuquerque, NM 87131. sumption into question (see Gordon, 1981, 1983; Gordon, Mowrer, McGinnis, \& McDermott, 1985; Mowrer \& Gordon, 1983; Weaver, 1988). These studies have indicated that contextual control over learned responding can be transferred from the learning context to a novel context by means of a cuing or reminder treatment. That is, if animals are trained in one context and are then given a cue to remind them of training in a novel context, the animals subsequently perform as if they had learned in the novel cuing context. In these studies, the cuing treatments have normally involved placing animals in the novel context and then confronting them with a brief presentation of some stimulus (usually a contextual stimulus) that had been present during the learning experience.

In an effort to explain these findings, we have followed a suggestion by Lewis (1979) that cuing treatments may cause animals to retrieve and actively reprocess their training memories. We have proposed that when cuing occurs in a novel environment, an organism may begin to process its representation of the training experience at the same time it is processing the incoming stimulation from the novel context. We have suggested that this joint processing may allow an organism to form associations between the novel contextual stimuli and elements of the training memory (e.g., the representation of the CS, the US, or the CS-US association as a whole). In effect, this proposal states that at the time of cuing, an animal can form new contextual associations of the type normally formed during learning. Thus, the cuing context comes to control performance in the same manner as the learning context originally does.

In the present experiments, we attempted to extend this line of research by determining whether or not a cuing treatment can be used to transfer the effects of CS preexposures from one context to another. It is well known that the course of Pavlovian conditioning can be slowed significantly by exposing an animal to the CS alone before conditioning trials commence (see Lubow, 1973). However, for this "latent inhibition" effect to occur, it appears to be necessary that the CS-alone exposures and the 
conditioning trials take place in the same context. When CS exposures and conditioning trials occur in different contexts, the development of conditioned responding appears to proceed normally (e.g., Channell \& Hall, 1983; Hall \& Channell, 1985; Hall \& Minor, 1984; Lovibond, Preston, \& Mackintosh, 1984). The question of interest in the present study is whether animals given CS exposures in one context and conditioning in another will show evidence of retarded acquisition, if they are reminded of the CS exposures in the conditioning context prior to the conditioning experience.

Examining the effects of cuing in a latent inhibition paradigm seems warranted on at least two grounds. First, in our previous studies, we have always trained animals to perform a given response in one context and have employed cuing treatments to enhance an animal's performance of that response in a novel context. In other words, we have always assumed that our animals have formed some specific association during training and that cuing has somehow enabled the animals to perform in accordance with this association in a novel context. In a latent inhibition paradigm, it is currently unclear whether an animal's initial experience (CS exposure) results in the formation of a specific association or whether this experience influences later conditioning via some nonassociative mechanism (see Lubow, Schnur, \& Rifkin, 1976; Mackintosh, 1975; Pearce, 1987; Pearce \& Hall, 1980; Wagner, 1978, 1981). Thus, it is unclear whether the presentation of a cue following CS exposures will function in the same manner as cuing after a conditioning experience.

A second impetus for these studies derives from a set of findings reported by Lovibond et al. (1984). Those results indicated that although CS exposure effects and conditioning effects both appear to be context-dependent, the mechanisms underlying this dependency may be different in these two cases. Specifically, they found that conditioning effects may transfer very well to a nontraining context as long as animals are familiar with the test context prior to testing, and as long as the training and testing contexts do not differ in some way that might affect an animal's perception of the CS. In contrast, they found that CS exposure effects are context-specific even when animals are familiar with the text contest and even when the context differences would not be expected to alter an organism's perception of the CS. These findings have not been supported by all subsequent studies (e.g., Hall \& Channell, 1985). However, these findings raise questions about the similarity of conditioning and CS exposure effects and how such effects might be modulated by cuing treatments.

\section{EXPERIMENT 1}

Previous studies have shown that latent inhibition effects can be reduced by giving animals CS preexposures and CS-US pairings in different contexts. The purpose of this experiment was to attempt to replicate this finding using a particular set of treatment parameters and contextual manipulations.

\section{Method}

Subjects. The subjects were 24 male and 24 female SpragueDawley rats, bred and reared at the University of New Mexico. They were housed in individual cages and were maintained under a 12:12 h light:dark cycle. At the time of experimental onset, all animals were between 60 and 90 days of age with body weights ranging from $200-290 \mathrm{~g}$. All behavioral testing was conducted at the same time each day during the light phase of the light-dark cycle.

Apparatus. Three identical activity-measuring apparatuses manufactured by Lafayette Instrument Co. (Model 86010) were employed. Each apparatus consisted of a platform, an enclosure, an activity-sensing transducer, and an amplifier. The platform of each apparatus was constructed of a fiberesin sheet measuring $30.5 \times 30.5 \mathrm{~cm}$. Mounted directly on top of the fiberesin platform was a $30.5 \times 30.5 \times 30.5 \mathrm{~cm}$ enclosure. The back and two side walls of each enclosure were made of aluminum; the front wall and hinged lid were made of clear Plexiglas. The right aluminum side wall of each chamber housed a food receptacle into which food pellets (45-mg Noyes brand) could be delivered by pellet dispensers (Lafayette Model 80200) located on the outside of the chamber. Each receptacle was located $19 \mathrm{~cm}$ above the fiberesin floor and $7.2 \mathrm{~cm}$ from the front Plexiglas wall. The Plexiglas lid of each chamber housed a speaker through which a $1200-\mathrm{Hz}, 90-\mathrm{dB}$ tone could be delivered.

Centered and mounted directly underneath the fiberesin platform was the activity-sensing transducer. This unit consisted of a permanent magnet suspended within a coil and was further anchored through the center of a $30.5 \times 30.5 \mathrm{~cm}$ sheet of Plexiglas and onto a second $30.5 \times 30.5 \mathrm{~cm}$ sheet of fiberesin that served as the overall baseboard of the apparatus. The activity-sensing platforms were then connected to the amplifiers which, in turn, were connected to two electromechanical data recorders (Lafayette Model 5804) located in an adjacent room. The amplifier of each apparatus provided a threshold control for varying the sensitivity of the platform to animal activity. Each threshold control was adjusted so that, over three trials, an average of 10 counts registered on the data recorders when a 20-g weight struck the center of the chamber floor from a distance of $30.5 \mathrm{~cm}$.

One experimental room was employed in the first experiment. This room was changed distinctively in terms of odor, lighting, noise level, and holding cages to make two different contexts, $A$ and $B$. Context $\mathrm{A}$ was designated as the conditioning context while both Context A and Context B were used for preexposure to the CS. The three activity chambers were always located in this room. To create Context A, the aluminum walls of each chamber were lined with gray cardboard. In addition, the fiberesin floor of each chamber was converted to one of wire mesh by placing a $30.5 \times 30.5 \mathrm{~cm}$ sheet of wire mesh on top of four (one located in each corner) Mason jar rings each measuring $7.62 \mathrm{~cm}$ in diameter. Three identical holding cages measuring $45 \times 23 \times 15 \mathrm{~cm}$ were used in Context $\mathrm{A}$. Each consisted of four opaque plastic walls and a wire lid. The floor, also made of opaque plastic, was covered with wood chips. In Context B, all four walls of each enclosure were lined with panel board having alternating 1-in. brown and white stripes. In addition, one of the jar lids under the wire mesh floor was replaced with a spearmint Stickup brand odorizer, which also measured $7.62 \mathrm{~cm}$ in diameter. Located directly below the three activity chambers was a speaker through which a continuous $80-\mathrm{dB}$ white-noise stimulus could be delivered in Context B. Three identical holding cages measuring $32 \times 16 \times 13.5 \mathrm{~cm}$ were used in Context B. Each was composed of four metal walls, a wire mesh floor, and a clear Plexiglas 
lid. A tray of wood chips was placed approximately $5 \mathrm{~cm}$ below the wire flooring and a spearmint Stickup brand odorizer was placed in one corner of the tray. The specific characteristics of the contexts are listed in Table 1.

Procedure. One week prior to experimental treatment, all animals were placed on a food-deprivation schedule designed to maintain them between $85 \%-90 \%$ of their free-feeding body weights. During this time, the animals were handled individually and tamed for 5 min every day, and were weighed after handling every other day. The animals continued to be weighed every other day throughout the experiment. Two days before beginning the experiment (i.e., Days 6 and 7 of deprivation), the animals were familiarized with the food pellets that were to serve as reinforcement during the conditioning phase. On each of these 2 days, the animals received and consumed $2045-\mathrm{mg}$ Noyes pellets in their home cage. On all days of deprivation, as well as on all treatment days, the animals were fed their daily allotment of food after the completion of each daily treatment.

On the first 2 days of the experiment, all animals were magazine trained in the activity chambers located in Context A. Magazine training involved delivering 10 reinforcements into the food receptacles in a 20 -min session. The mean intertrial interval (ITI) was $2.0 \mathrm{~min}$ and each reinforcing event consisted of two pellets delivered one at a time, with a 0.50 -sec interval between the two. At the end of this training, the subjects were randomly assigned to one of three groups consisting of 8 males and 8 females each.

On the next 4 days, animals in the SAME and DIFF groups were preexposed to the tone stimulus that was later to serve as the CS. On each of these days, the animals in Group SAME were taken to Context $A$ and placed in the holding cages for $30 \mathrm{sec}$. They were then placed in the activity chambers and given 1010 -sec tone-alone presentations. The session duration was $50 \mathrm{~min}$ and the mean ITI was $5.0 \mathrm{~min}$. The animals in Group DIFF received the exact same treatment, except that it occurred in Context B. In this context, the background white noise was initiated after all animals had been removed from the holding cages and placed in the activity chambers. On these 4 days, the animals in Group CONTROL remained in the animal colony and were simply weighed and/or handled.

On the next 2 days, all animals were given tone-food pairings in Context A. On each of these days, the animals were taken to Context $A$ and placed in the holding cages for $30 \mathrm{sec}$. They were then placed in the activity chambers and given four, 10-sec tone presentations, each followed immediately by food reinforcement. As in the magazine-training phase, reinforcement consisted of two pellets separated by a 0.50 -sec interval. The session duration was $50.0 \mathrm{~min}$ and the mean ITI was $12.0 \mathrm{~min}$.

In both the CS preexposure and the conditioning phases, general activity was the response measured. Specifically, the number of activity counts occurring during and $30 \mathrm{sec}$ before each tone presentation were recorded and were converted to the number of activity counts per minute. To assess activity changes in the presence of the tone, a ratio was obtained for each animal by dividing the number of activity counts per minute during the CS by the number of activity counts per minute during the pre-CS period. This ratio was then transformed to a $\log$ activity ratio $[\log 10(x+1)]$ such that an activity ratio of 0.30 indicated that activity was equivalent during the CS and the pre-CS periods, whereas a ratio greater than 0.30 indicated that there was more activity during the CS than the preCS period.

\section{Results and Discussion}

The left panel of Figure 1 shows mean log activity ratios for Groups SAME and DIFF during the last eight trials (i.e., Trials 33-40) of the CS-alone preexposure phase. A repeated measures analysis of variance (ANOVA) performed on these data revealed that there were no significant differences between groups $[F(1,30)<1]$ or across trials $[F(7,210)<]$. Likewise, the group $\times$ trials interaction was not significant $[F(7,210)<1.07]$. In this and all subsequent analyses, a rejection region of .05 was employed. These preliminary results indicate that activity to the tone was relatively equivalent for the groups prior to the conditioning phase.

The right panel of Figure 1 shows mean log activity ratios for all groups during the eight conditioning trials. A repeated measures ANOVA performed on these data revealed significant effects of group $[F(2,45)=3.73]$ and trials $[F(7,315)=5.48]$, as well as a significant interaction of these two variables $[F(14,315)=2.03]$. Subsequent simple-effect analyses revealed that it was on Trials $2[F(2,45)=3.56], 6[F(2,45)=3.75]$, and $8[F(2,45)=$ $4.93]$ that the groups differed significantly. Importantly, no differences among group means were detected on Trial 1. As is suggested by Figure 1, activity in the presence of the tone was equivalent across groups prior to receiving food reinforcement at the end of this first conditioning trial.

To further analyze differences among the individual group means on Trials 2, 6, and 8, the Newman-Keuls statistic (Kirk, 1968) was employed. This test revealed that Group DIFF displayed significantly more activity to the tone than did the other two groups on the second conditioning trial. Given that these group differences were not maintained across the next few trials, this rapid in-

Table 1

Characteristics of Experimental Treatment Contexts

\begin{tabular}{|c|c|c|c|}
\hline & \multicolumn{3}{|c|}{ Context } \\
\hline & $\mathbf{A}$ & B & C \\
\hline Dimensions & $16.0 \times 6.5 \mathrm{ft}$ & $16.0 \times 6.5 \mathrm{ft}$ & $16.0 \times 6.5 \mathrm{ft}$ \\
\hline Lighting & Dim, 0.17 & Bright, 1.0 & Moderate, 0.60 \\
\hline Odor (room deodorizer) & None & Spearmint & Honeysuckle \\
\hline Noise Level & Fan, 63-65 dB & White noise, $80 \mathrm{~dB}$ & None $45-50 \mathrm{~dB}$ \\
\hline Gloves & None & Cotton & Suede \\
\hline Holding Cage & Plastic & Metal & Wire mesh \\
\hline Activity Chamber & $\begin{array}{l}3 \text { gray walls } \\
1 \text { clear Plexiglas wall and lid } \\
\text { wire-mesh floor }\end{array}$ & $\begin{array}{l}\text { Striped walls } \\
\text { clear Plexiglas lid } \\
\text { wire-mesh floor }\end{array}$ & None \\
\hline
\end{tabular}




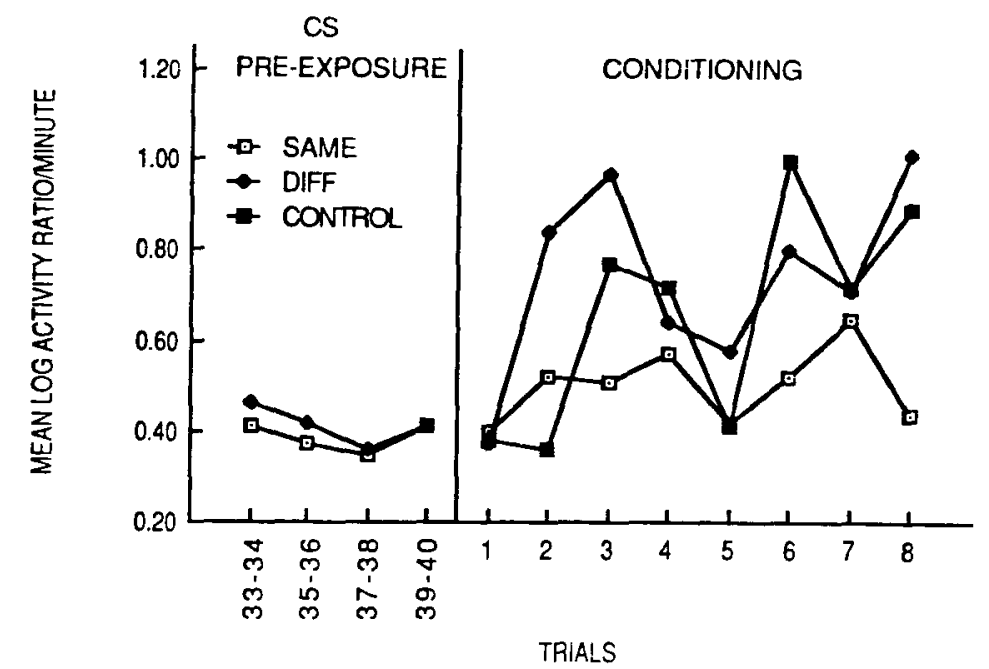

Figure 1. Mean log activity ratios to the tone CS during the last eight trials of the CS-alone preexposure phase (left panel) and during the eight trials of the CS-US conditioning phase (right panel) in Experiment 1.

crease in activity may simply have been due to four very extreme scores in Group DIFF. By Trials 6 and 8, Groups DIFF and CONTROL were displaying fairly equivalent amounts of activity in the presence of the tone. Although both groups appeared to be more active during the tone than was Group SAME, it was only on Trial 8 that Group DIFF significantly differed from Group SAME. Group CONTROL displayed significantly more activity than did Group SAME on both trials.

Thus, it appears that the tone paired with food generated more activity for the groups that had received their CS preexposure in a different context (DIFF) or had received no CS preexposure (CONTROL). This notion was further confirmed by comparing Trials 1 and 8 within each group. As is suggested by Figure 1, the reinforced tone CS evoked much greater activity on the last conditioning trial than on the first for both Group CONTROL $[t(30)=3.06]$ and Group DIFF $[t(30)=4.00]$. On the other hand, such increased activity was not exhibited by Group SAME $[t(30)=0.31, p>.05]$.

The results of this experiment serve to establish effective parameters for producing a latent inhibition effect within this appetitive activity conditioning paradigm. In addition, it provides an effective manipulation by which to attenuate this effect, namely, changing the context between the CS preexposure and conditioning phases.

\section{EXPERIMENT 2}

In Experiment 1, we found that latent inhibition effects can be diminished by giving CS preexposures and conditioning trials in different contexts. The purpose of the present experiment was to determine whether latent inhibition effects can be restored by means of a cuing treatment. Specifically, if rats are reminded of CS preexposures in the novel conditioning context before CS-US pairings be- gin, will latent inhibition be exhibited in the novel conditioning context?

\section{Method}

Subjects and Apparatus. Forty-two female rats similar to those employed in the previous experiment served as subjects. The conditioning apparatuses and experimental contexts (A and B) employed in Experiment 1 were also utilized in Experiment 2.

Procedure. All pretraining, magazine training, CS preexposure, and conditioning procedures were carried out precisely as in Experiment 1 . In addition, at the end of magazine training, the subjects were assigned randomly to one of three groups consisting of 14 rats each.

The first two groups in the present experiment, SAME and DIFF, were treated identically to Groups SAME and DIFF in Experiment 1 . The third group, WN(A), was treated identically to Group DIFF, with one exception. The animals in Group WN(A) received a reminder treatment in Context $\mathbf{A}$ prior to being conditioned there. This reminder, or cuing, treatment consisted of bringing the animals to Context $A$ and placing them in the holding cage for $30 \mathrm{sec}$. At the end of this time, the white noise, which had been constantly present in Context B, was presented for $15 \mathrm{sec}$. The animals remained in the holding cages for an additional $3.5 \mathrm{~min}$ and were then placed in the activity chamber for conditioning.

\section{Results and Discussion}

The left panel of Figure 2 shows mean log activity ratios for all groups during the last eight trials (i.e., Trials 33-40) of the CS-alone preexposure phase. A repeated measures ANOVA performed on these data revealed that there were no significant differences between groups $[F(2,39)<1]$ or across trials $[F(7,273)<1]$. Likewise, the group $\times$ trials interaction was not significant $[F(14,273)=1.02]$. Thus, as in Experiment 1, activity to the tone was relatively equivalent across groups prior to the conditioning phase.

The right panel of Figure 2 shows mean log activity ratios during the eight conditioning trials. A repeated 


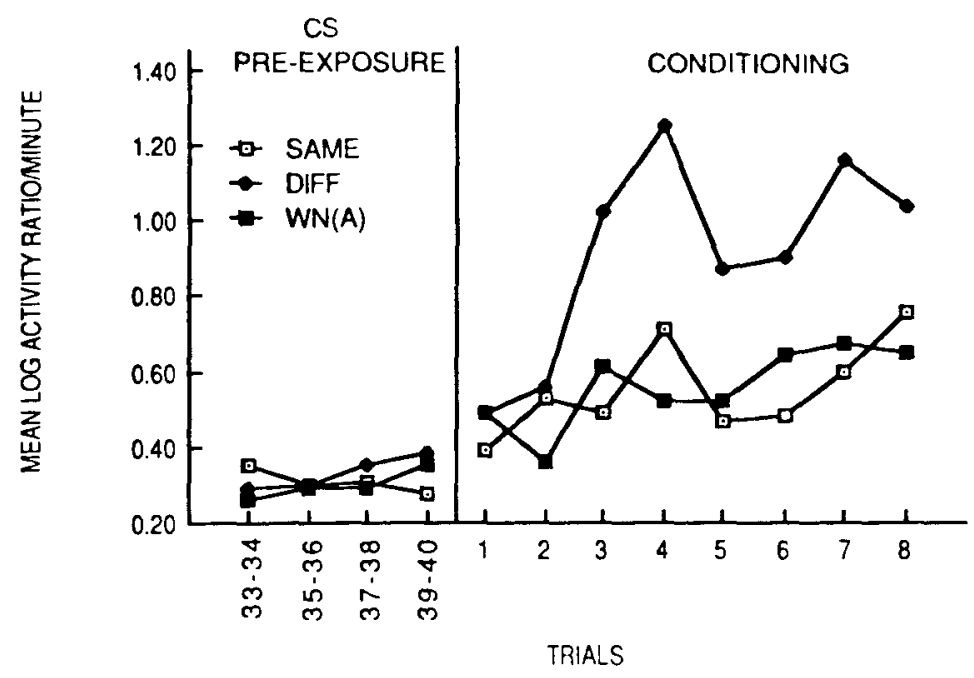

Figure 2. Mean log activity ratios to the tone CS during the last eight trials of the CS-alone preexposure phase (left panel) and during the eight trials of the CS-US conditioning phase (right panel) in Experiment 2.

measures ANOVA performed on these data revealed significant effects of group $[F(2,39)=18.43]$ and trials $[F(7,273)=6.54]$, as well as a significant interaction $[F(14,273)=1.82]$. Subsequent simple-effect analyses revealed that the groups differed significantly on Trials $3[F(2,39)=7.91], 4[F(2,39)=14.50], 5[F(2,39)=$ $3.83], 6[F(2,39)=3.42], 7[F(2,39)=7.29]$, and 8 $[F(2,39)=3.23]$. As in the first experiment, no differences among group means were detected on the first conditioning trial, thus indicating that activity in the presence of the tone was equivalent across groups prior to receiving food reinforcement. According to the Newman-Keuls statistic, Group DIFF displayed significantly more activity to the tone than did the other two groups on all trials except the eighth. On this trial, only Group DIFF and WN(A) continued to differ; Group SAME did not differ from either Group DIFF or Group WN(A).

These results indicate that although Group DIFF was showing greater conditioning to the tone across Trials 3-7 relative to the other two groups, by Trial 8, Group SAME had started to show signs of conditioning. This notion was further confirmed by comparing Trials 1 and 8 within each group. As is suggested by Figure 2, the reinforced tone CS evoked greater activity on the last conditioning trial than on the first for both Group DIFF $[t(26)=3.36]$ and Group SAME $[t(26)=2.71]$. On the other hand, such increased activity was not evidenced by Group WN(A) $[t(26)=1.32, p>.05]$.

Thus, as in Experiment 1, pairing the tone with food generated much more activity for the group that had received its CS preexposure in a different context (DIFF) than for the group that had received its CS preexposure in the conditioning context (SAME). Importantly, the group that received cuing [WN(A)] performed very much like Group SAME, except on Trial 8. The discrepancy on this trial may have been due to the latent inhibition effect dissipating more rapidly for Group SAME than for Group WN(A).

The results of this experiment not only replicate the findings of Experiment 1, but also suggest that cuing is an effective manipulation to transfer the latent inhibition effect from one context to another.

\section{EXPERIMENT 3}

Experiment 2 showed that the effects of CS preexposure can be transferred from one context to another by means of a cuing treatment. The purpose of Experiment 3 was to include certain control conditions that would allow us to interpret the effects of the cuing treatment more explicitly. In particular, must the cuing treatment be designed specifically to remind animals of a CS-alone exposure in order for the latent inhibition effect to be restored? Also, must the cuing treatment occur in the conditioning context in order to be effective? The answers to these questions are critical if we are to identify the mechanism by which cuing affects conditioned responding.

\section{Method}

Subjects and Apparatus. Fifty female rats similar to those employed in the previous experiments served as subjects. The conditioning apparatuses and experimental contexts (A and B) employed in Experiment 2 were also utilized in Experiment 3. In addition, a second room was used to provide a third context, $C$. As with the two previously used contexts, Context $C$ differed distinctively in terms of color, lighting, noise level, and type of holding cages. Since this room was used only as cuing context, no conditioning chambers were located in it. Three identical holding cages measuring $38 \times 22 \times 19 \mathrm{~cm}$ were used in Context $\mathrm{C}$. Each enclosure was constructed entirely from wire mesh with one endwall being hinged so that it served as a door. A tray of wood chips was placed ap- 
proximately $5 \mathrm{~cm}$ below the wire mesh flooring and a honeysuckle Stickup brand odorizer was placed in one corner of the tray. (See Table 1 for the specific characteristics of this room.)

Procedure. All pretraining, magazine training, CS preexposure, and conditioning procedures were carried out precisely as in the previous two experiments. In addition, unless otherwise specified, the cuing procedure was carried out just as in Experiment 2. At the end of the magazine training, the subjects were randomly assigned to one of five groups consisting of 10 rats each.

The first two groups in the present experiment, DIFF and WN(A), were treated identically to Groups DIFF and WN(A) in Experiment 2 . The three remaining groups were treated identically to Group WN(A), except for one procedural difference within each treatment condition. The third group, WN(C), was employed to demonstrate that the cuing treatment is effective only when it is administered in the relevant conditioning context. As such, the animals in this group received their reminder treatment in Context $\mathrm{C}$ instead of in Context $\mathrm{A}$. In this case, the animals were taken to Context $\mathrm{C}$ and placed in holding cages for $30 \mathrm{sec}$. At the end of this time, the white noise was presented for $15 \mathrm{sec}$. The animals remained in the holding cages for an additional $3.5 \mathrm{~min}$ in Context $\mathrm{C}$ and were then transported to Context $A$ and placed in the activity chambers for conditioning. The fourth group, NoWN-WN(A), was employed in order to show that the cuing treatment is effective only when the cuing stimulus has been part of the overall preexposure experience. Thus, for these animals, the white noise was not present during the preexposure phase. The fifth group, COND-WN(A), was included to demonstate that cuing does not, in and of itself, attenuate activity to the CS. That is, this group was included to demonstrate that the outcome of the cuing treatment depends on the previous experience with the cuing stimulus. The animals in this group were given CS-US conditioning sessions insead of CSalone preexposure sessions. That is, for these animals, the 10,10-sec tone presentations that occurred in each session were followed immediately by the two-pellet reinforcement event.

\section{Results and Discussion}

The left panel of Figure 3 shows mean log activity ratios to the tone during the last eight trials of the CS-alone preexposure phase for Groups DIFF, WN(A), WN(C), and NoWN-WN(A), and the last eight trials of the CS-US conditioning phase for Group COND-WN(A). A one-way ANOVA on the Trial $\mathbf{4 0}$ data revealed a significant effect of group $[F(4,45)=6.48]$. The Newman-Keuls procedure revealed that the activity displayed by Group COND-WN(A) was reliably greater than for any other group on this trial $(p<.05)$. No other comparisons reached significance, indicating that activity in the presence of the tone was equivalent among the nonreinforced groups prior to the conditioning phase.

The right panel of Figure 3 shows mean log activity ratios to the tone during the eight conditioning trials in Context A. A repeated measures ANOVA performed on these conditioning trials revealed significant effects of group $[F(4,45)=8.59]$ and trials $[F(7,315)=13.78]$, as well as a significant group $\times$ trials interaction $[F(28,315)=1.55]$. An analysis of this interaction indicated that there were significant differences among the groups on Trials $1[F(4,45)=4.72], 2[F(4,45)=2.66]$, $7[F(4,45)=3.69]$, and $8[F(4,45)=6.80]$. The Newman-Keuls procedure revealed that the activity displayed by Group COND-WN(A) on Trial 1 was significantly greater $(p<.05)$ than that displayed by any of the other four groups. Importantly, all other comparisons among the four remaining groups failed to reach significance $(p>.05)$, indicating that activity in the presence of the tone was equivalent across these groups before they received food reinforcement. The fact that Group COND-WN(A) maintained a higher level of activity suggests that cuing may have reversed the performance decrement that normally occurs after a contextual shift.

On Trial 2, the Newman-Keuls test revealed a significant difference between the means of Groups CONDWN(A) and WN(A) $(p<.05)$. All other comparisons for this trial failed to reach significance, suggesting that

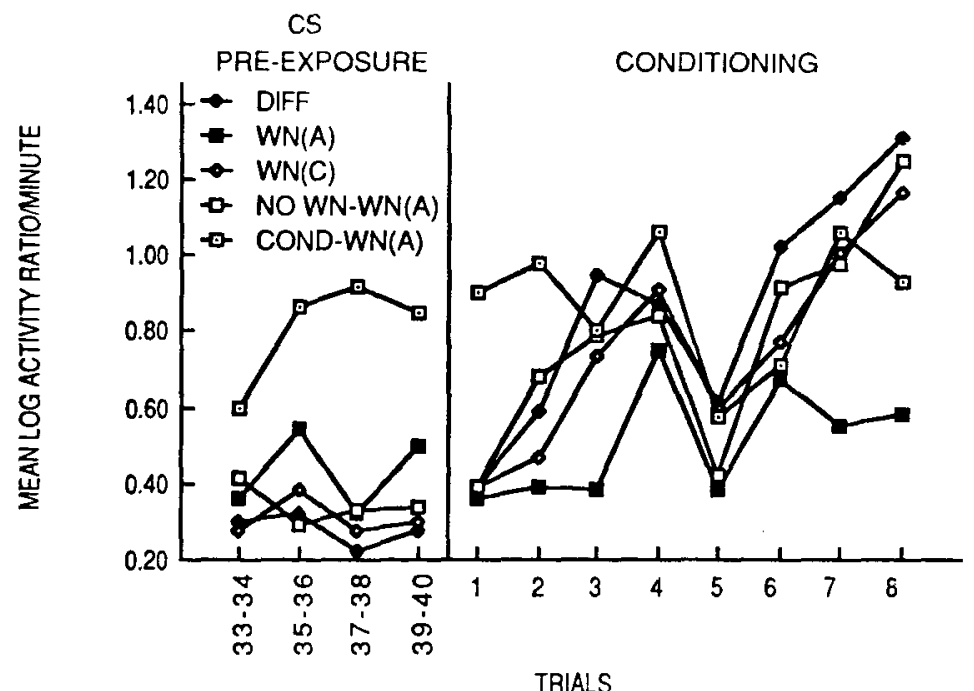

Figure 3. Mean log activity ratios to the tone CS during the last eight trials of the CS-alone preexposure phase (left panel) and during the eight trials of the CS-US conditioning phase (right panel) in Experiment 3. 
the activity displayed by the three remaining groups was somewhere in between the means of the two extreme groups.

By Trials 7 and 8 , the activity displayed by Groups COND-WN(A), DIFF, WN(C), and NoWN-WN(A) was significantly greater $(p<.05)$ than that displayed by Group WN(A). All other comparisons failed to reach significance, indicating that, over trials, activity increased more for the groups that received their CS preexposure in a different context (DIFF) or were administered irrelevant cuing treatments [WN(C) and no WN-WN(A)]. On the other hand, although activity increased, it remained minimal for Group WN(A), which received its cuing treatment in the relevant context. That this phenomenon is not simply due to some attenuating effect of cuing is evidenced by Group COND-WN(A). In particular, after being cued, this group maintained its higher level of responding when it was shifted from Context $B$ to Context $A$ and continued to perform at an asymptotic level. These conclusions were further confirmed by comparing Trials 1 and 8 within each group. As is suggested by Figure 3, the reinforced tone CS evoked much more activity on the last conditioning trial than on the first for Groups DIFF $[t(18)=5.48]$, $\mathrm{WN}(\mathrm{C})[t(18)=8.54]$, and NoWN-WN(A) $[t(18)=$ 5.08]. Activity levels did increase from Trial 1 to Trial 8 for Group WN(A), although not quite as much $[t(18)=$ 2.37], but it remained relatively stable for Group COND$\mathrm{WN}(\mathrm{A})[t(18)=2.10, p>.05]$.

\section{GENERAL DISCUSSION}

The present findings confirm previous reports that the effects of CS preexposures tend to be context-specific (e.g., Channell \& Hall, 1983; Hall \& Channell, 1985). Experiment 1 demonstrated that when CS preexposure and CS-US pairings occur in the same context, conditioning is slowed significantly. However, this retardation of conditioning is not evident when preexposures and conditioning trials occur in different contexts.

Importantly, the present experiments also demonstate that cuing treatments can serve to transfer CS preexposure effects from one context to another. Thus, animals given CS preexposures and conditioning trials in different contexts do exhibit slowed conditioning, if they are reminded of the preexposures in the conditioning context before CS-US pairings begin. This reinstatement of latent inhibition effects was shown to occur only when the cuing treatment involved a stimulus that was present during CSalone presentations and only when the cuing treatment was administered in the conditioning context. That is, cuing was effective only when the cue could have served to remind the animals of the CS preexposure while they were in the conditioning context.

These results indicate that CS preexposure effects, like the effects of a conditioning experience, can be transferred to a novel context via the presentation of a cuing treatment. The question left unanswered by these results is what mechanism might underlie this cue-induced transfer effect. In the case of conditioning effects, many researchers have assumed that at the time of learning, contextual stimuli enter into associations with various elements of the conditioning situation (e.g., the CS, the US, or the CS-US association). These researchers have proposed that as a result of such associations, the learning context becomes capable of either eliciting a CR on its own (e.g., Rescorla \& Wagner, 1972) or retrieving the CS-US association that is formed (e.g., Nadel $\&$ Willner, 1980; Spear, 1973). According to both views, the contextual associations, developed lead to greater responding in the learning context than in a novel test environment.

To explain the cue-induced transfer of conditioning effects to a novel context, we have proposed that cuing results in the activation of an animal's training memory, and that such activation allows an animal to form new associations between the cuing context and representations of the training stimuli (see Gordon, 1983). In effect, we have suggested that at the time of cuing, contextual stimuli enter into the same kinds of associations that are normally formed at the time of learning. Thus, the novel cuing context either becomes capable of eliciting a CR on its own or acquires the capacity to retrieve the CS-US association formed during learning.

The applicability of this interpretation to the present results depends on the view one adopts concerning the nature of latent inhibition and why these effects are normally context-specific. For example, one explanation of latent inhibition effects is that during CS-alone exposures, animals learn that the CS is irrelevant or that it signals nothing of consequence (e.g., Baker \& Mackintosh, 1977). It is assumed that such learning then makes it more difficult for an organism to learn that the CS does signal an important event (the US) at the time of conditioning. Implicit in this view is the idea that, at the time of CS preexposure, an animal forms some representation of the CS as a signal for no important consequence. Presumably this "irrelevant CS" representation is encoded along with the exposure context so that the context becomes capable of retrieving the CS representation. When animals are conditioned outside the exposure context, they fail to retrieve the irrelevant CS memory and conditioning proceeds normally.

Obviously, if one adopts this explanation of latent inhibition effects, the cuing hypothesis we have used previously applies rather easily to the present results. We would simply assume that when an animal is cued in a novel context, the representation of the irrelevant CS is activated in the presence of the new contextual stimuli. This would allow the animal to reencode the CS representation along with the novel context, and the novel context would later function to retrieve the CS representation during conditioning.

A second interpretation of latent inhibition effects, proposed by Wagner $(1978,1981)$, is based on the idea that 
during CS exposures, an animal forms an association between the CS and the exposure context. As a result of this association, the exposure context becomes capable of "priming," or activating, the CS representation. According to Wagner's more general conditioning theory, the associability of any stimulus is reduced if some representation of that stimulus is already primed or active at the time that stimulus occurs (see Wagner, 1981). Thus, when an animal is placed in the exposure context for its conditioning trials, the representation of the CS is activated by the context and the probability that the CS will become associated with the US is lessened. When conditioning occurs outside the exposure context, the CS representation is not activated prior to CS occurrence and conditioning occurs normally.

If we accept this view of why latent inhibition is contextspecific, then the current results suggest that cuing somehow enables the cuing context to activate the CS representation, just as the exposure context originally does. Again, it is possible to use the aforementioned cuing hypothesis to explain how the cuing context might acquire this capacity. We would simply suggest that the cuing treatment activates the CS representation and allows for an association to be formed between the CS and the novel context. This new association would then enable the novel context to prime the CS representation at the time of conditioning. Although such an interpretation would seem to be viable given Wagner's explanation for latent inhibition, there is one problem with using such an interpretation given the constraints of Wagner's conditioning model.

We are assuming that at the time of cuing, an animal's $\mathrm{CS}$ representation is being primed or retrieved via the cuing treatment, and that this primed representation is then being associated with the new contextual stimuli that are present. According to Wagner's model, however, whenever a representation is retrieved, that representation enters a peripheral state of activation (State A2 in his model). Thus, at the time of cuing, Wagner's model would assume that the CS representation resides in this peripheral active state. On the other hand, Wagner's model assumes that when an animal perceives a stimulus for the first time, that stimulus becomes represented in a focal state of activation, termed A1.

According to Wagner's model, excitatory associations can be formed between stimuli only to the extent that they are represented simultaneously in the focal active state. Stimuli represented in the peripheral State A2 cannot enter into excitatory associations with any other stimulus. Thus, Wagner's model clearly prohibits the formation of an excitatory association between a retrieved representation and novel stimuli that are being perceived for the first time. This, of course, is precisely the type of association we are suggesting might be formed at the time of cuing.

In effect, if we adopt Wagner's model of conditioning and his explanation for latent inhibition, we are forced to propose an alternative mechanism for the present cuing effect. One possibility is that cuing may result in a direct association between the cuing stimulus (a stimulus from the preexposure context) and the novel cuing context. During conditioning, the cuing context might then activate a representation of the cuing stimulus, which in turn could prime the representation of the preexposed CS. This indirect priming of the CS representation should still retard the conditioning process. Although such an account is admittedly complex, it is consistent with Wagner's explanatory framework.

Finally, some explanations of latent inhibition suggest that during CS preexposures, the CS loses salience as an animal's attention to that stimulus wanes (see Mackintosh, 1975; Pearce \& Hall, 1980). It is this reduction in salience that is assumed to be responsible for the poor conditioning that later occurs. Although many such accounts provide no clear explanation for why latent inhibition might be context-specific, a recent model proposed by Pearce (1987) does speak to this issue.

Pearce hypothesizes that whenever a CS is presented in a given context, the $C S$ and the context combine to form a configural stimulus. Thus, during CS-alone exposures, the entire configural stimulus (CS + context) loses salience and becomes less associable. When conditioning to the CS occurs in a novel context, the configural stimulus is altered and the new configural stimulus is more salient than the preexposed stimulus. As a result of this increased salience, latent inhibition effects are reduced when conditioning occurs outside the preexposure context.

Basically, this account suggests that when animals are given CS exposures in one context and are then conditioned in another, a generalization decrement occurs. The effects of preexposure fail to generalize to the conditioning phase because of the change in the preexposed configural stimulus. To explain the present cuing effects within this framework, it is necessary to assume that cuing somehow increases the similarity between the preexposure and the conditioning contexts. At this point, we can only speculate as to how cuing might promote such an increase in similarity. One possibility is that when a stimulus from the preexposure context is presented in the conditioning context, an animal may encode these stimuli together. Thus, the animal's representation of the conditioning context would include some representation of the cuing stimulus. In effect, the cuing stimulus would then become a common element in the animal's representations of the two contexts. According to Pearce's model, the more common elements two representations share, the more similar those representations are. Thus, cuing might reduce a generalization decrement by increasing the similarity of the preexposure and conditioning contexts.

The present results are clear in showing that a cuing treatment can reduce the context-specificity of latent inhibition effects. It is equally clear, however, that we can only speculate at the present time about the mechanisms that govern this effect. As we learn more about the nature of latent inhibition and contextual influences over that phenomenon, we should reach a better understanding of 
the current cuing results. A more complete understanding of latent inhibition might also enable us to formulate a unified account of cuing effects that can be applied to both conditioning and CS preexposure situations.

\section{REFERENCES}

BaKer, A. G., Mackintosh, N. J. (1977). Excitatory and inhibitory conditioning following uncorrelated presentations of CS and UCS. Animal Learning \& Behavior, 5, 315-319.

Balsam, P. D., \& Tomie, A. (1985). Context and learning. Hillsdale, NJ: Erlbaum.

Channell, S., Hall, G. (1983). Contextual effects in latent inhibition with an appetitive conditioning procedure. Animal Learning \& Behavior, 11, 67-74.

GORDON, W. C. (1981). Mechanisms of cue-induced retention enhancement. In N. E. Spear \& R. R. Miller (Eds.), Information processing in animals: Memory mechanisms (pp. 319-339). Hillsdale, NJ: Erlbaum.

GoRDON, W. C. (1983). The malleability of memory in animals. In R. L. Mellgren (Ed.), Animal cognition and behavior (pp. 399-426). Amsterdam: North-Holland.

Gordon, W. C., Mowrer, R. R., McGinnis, C. P., \& McDermott, M. J. (1985). Cue-induced memory interference in the rat. Bulletin of the Psychonomic Society, 23, 233-236.

Hall, G., Channell, S. (1985). Differential effects of contextual change on latent inhibition and on the habituation of an orienting response. Journal of Experimental Psychology: Animal Behavior Processes, 11, 470-481.

HALL, G., \&inor, H. (1984). A search for context-stimulus associations in latent inhibition. Quarterly Joumal of Experimental Psychology, 36B, 145-169.

KIRK, R. E. (1968). Experimental design: Procedures for the behavioral sciences. Belmont, CA: Brooks-Cole.

LEWIs, D. J. (1979). Psychobiology of active and inactive memory. Psychological Bulletin, 86, 1054-1083.

Lovibond, P. F., Preston, G. C., Mackintosh, N. J. (1984). Context specificity of conditioning, extinction, and latent inhibition. Journal of Experimental Psychology: Animal Behavior Processes, 10, 360-375.
Luвow, R. E. (1973). Latent inhibition. Psychological Bulletin, 79. $398-407$

Lubow, R. E., SchnuR, P., Rifkin, B. (1976). Latent inhibition and conditioned attention theory. Journal of Experimental Psychology: Animal Behavior Processes, 2, 163-174.

MACkintosh, N. J. (1975). A theory of attention: Variations in the associability of stimuli with reinforcement. Psychological Review, 82 . 276-298.

MEDIN, D. L. (1975). A theory of context in discrimination learning. In G. H. Bower (Ed.), The psychology of learning and motivation (Vol. 9, pp. 263-314). New York: Academic Press.

Mowrer, R. R., Gordon, W. C. (1983). Effects of cuing in an "irrelevant" context. Animal Learning \& Behavior, 11, 401-406.

NAdel, L., Willner, J. (1980). Context and conditioning: A place for space. Physiological Psychology, 8, 218-228.

Pearce, J. M. (1987). A model for stimulus generalization in Pavlovian conditioning. Psychological Review, 94, 61-73.

Pearce, J. M., \& Hall, G. (1980). A model for Pavlovian learning: Variations in the effectiveness of conditioned but not unconditioned stimuli. Psychological Review, 87, 532-552.

Rescorla, R. A., W Waner, A. R. (1972). A theory of Pavlovian conditioning: Variations in the effectiveness of reinforcement and nonreinforcement. In A. H. Black \& W. F. Prokasy (Eds.), Classical conditioning: 2. Current theory and research (pp. 64-99). New York: Appleton-Century-Crofts.

SPEAR, N. E. (1973). Retrieval of memory in animals. Psychological Review, 80, 163-194.

WAGNER, A. R. (1978). Expectancies and the priming of STM. In S. H. Hulse, H. Fowler, \& W. K. Honig (Eds.), Cognitive processes in animal behavior (pp. 177-209). Hillsdale, NJ: Erlbaum.

WAGNER, A. R. (1981). SOP: A model of automatic memory processing in animal behavior. In N. E. Spear \& R. R. Miller (Eds.), Information processing in animals: Memory mechanisms (pp. 5-47). Hillsdale, NJ: Erlbaum.

WEAVER, M. S. (1988). Conditions under which the context-shift effect is eliminated within the blocking paradigm. Unpublished doctoral dissertation, University of New Mexico, Albuquerque.

(Manuscript received September 16, 1988; revision accepted for publication March 9, 1989.) 